Article

\title{
Simplified Iohexol-Based Method for Measurement of Glomerular Filtration Rate in Goats and Pigs
}

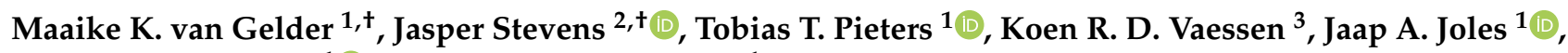 \\ Marianne C. Verhaar ${ }^{1}(1)$ and Karin G. F. Gerritsen ${ }^{1, *}$ \\ 1 Department of Nephrology and Hypertension, University Medical Center Utrecht, Heidelberglaan 100, \\ 3584 CX Utrecht, The Netherlands; m.k.vangelder-7@umcutrecht.nl (M.K.v.G.); \\ T.T.Pieters-4@umcutrecht.nl (T.T.P.); J.A.Joles@umcutrecht.nl (J.A.J.); M.C.Verhaar@umcutrecht.nl (M.C.V.) \\ 2 Department of Clinical Pharmacy and Pharmacology, University Medical Center Groningen, \\ University of Groningen, Hanzeplein 1, 9713 GZ Groningen, The Netherlands; j.stevens@umcg.nl \\ 3 Central Laboratory Animal Research Facility, Utrecht University, Heidelberglaan 8, \\ 3584 CS Utrecht, The Netherlands; K.R.D.Vaessen@uu.nl \\ * Correspondence: K.G.F.Gerritsen@umcutrecht.nl \\ + Both authors contributed equally.
}

check for updates

Citation: van Gelder, M.K.; Stevens, J.; Pieters, T.T.; Vaessen, K.R.D.; Joles, J.A.; Verhaar, M.C.; Gerritsen, K.G.F. Simplified Iohexol-Based Method for Measurement of Glomerular Filtration Rate in Goats and Pigs. Biology 2021, 10, 461. https:// doi.org/10.3390/biology10060461

Academic Editor: Isabel R. Dias

Received: 6 May 2021

Accepted: 15 May 2021

Published: 23 May 2021

Publisher's Note: MDPI stays neutral with regard to jurisdictional claims in published maps and institutional affiliations.

Copyright: (c) 2021 by the authors. Licensee MDPI, Basel, Switzerland. This article is an open access article distributed under the terms and conditions of the Creative Commons Attribution (CC BY) license (https:/ / creativecommons.org/licenses/by/ $4.0 /)$.
Simple Summary: To improve the treatment of patients with kidney disease, new therapies are being developed. Before being used on humans, such therapies need to be tested on animals with kidney disease because reduced kidney function may influence the safety and efficacy of the treatment. Using large animals for this purpose is important because they tolerate frequent blood sampling, which allows for repeated monitoring. Goats seem particularly suitable for the evaluation of novel hemodialysis therapies since they are docile, have easily accessible neck veins to obtain blood access and body weights comparable with humans. Currently, no simple method is available to measure kidney function in goats (with or without impaired kidney function). Therefore, we developed a simple method to measure the kidney function in goats and pigs, which is based on a single injection of iohexol and requires three blood samples. Subsequently, kidney function can be calculated using a formula derived from pharmacokinetic modelling. The measurement of kidney function using our simplified method is relatively easy to perform, reduces total blood sampling and eliminates the need for an indwelling bladder catheter as compared to existing methods that require continuous infusion of a substance and timed urine collection.

Abstract: The preclinical evaluation of novel therapies for chronic kidney disease requires a simple method for the assessment of kidney function in a uremic large animal model. An intravenous bolus of iohexol was administered to goats ( 13 measurements in $n=3$ goats) and pigs ( 23 measurements in $n=5$ pigs) before and after induction of kidney failure, followed by frequent blood sampling up to 1440 min. Plasma clearance (CL) was estimated by a nonlinear mixed-effects model (CLNLME) and by a one-compartmental pharmacokinetic disposition model using iohexol plasma concentrations during the terminal elimination phase $\left(\mathrm{CL}_{1 \mathrm{CMT}}\right)$. A simple method $\left(\mathrm{CL}_{\mathrm{SM}}\right)$ for the calculation of plasma clearance was developed based on the most appropriate relationship between $\mathrm{CL}_{\mathrm{NLME}}$ and $\mathrm{CL}_{1 \mathrm{CMT}}$. $\mathrm{CL}_{\mathrm{SM}}$ and $\mathrm{CL}_{\mathrm{NLME}}$ showed good agreement $\left(\mathrm{CL}_{\mathrm{NLME}} / \mathrm{CL}_{\mathrm{SM}}\right.$ ratio: $1.00 \pm 0.07$; bias: $0.03 \pm 1.64 \mathrm{~mL} / \mathrm{min}$; precision $\mathrm{CL}_{\mathrm{SM}}$ and $\mathrm{CL}_{\mathrm{NLME}}: 80.9 \%$ and $80.7 \%$, respectively; the percentage of $\mathrm{CL}_{\mathrm{SM}}$ estimates falling within $\pm 30 \%$ (P30) or $\pm 10 \%$ (P10) of $\mathrm{CL}_{\mathrm{NLME}}: 53 \%$ and $12 \%$, respectively). For mGFR $_{\text {NLME }}$ vs. mGFR $_{S M}$, bias was $-0.25 \pm 2.24$ and precision was $49.2 \%$ and $53.6 \%$, respectively, P30 and P10 for mGFR based on $\mathrm{CL}_{\mathrm{SM}}$ were $71 \%$ and $24 \%$, respectively. A simple method for measurement of GFR in healthy and uremic goats and pigs was successfully developed, which eliminates the need for continuous infusion of an exogenous marker, urine collection and frequent blood sampling.

Keywords: glomerular filtration rate; iohexol; plasma clearance; uremic animal model; goat; chronic kidney disease 


\section{Introduction}

Chronic kidney disease (CKD) is an important health care problem affecting approximately $10-16 \%$ of the population worldwide [1,2]. To improve the outcome of patients with CKD, there is a need for novel therapies. The preclinical evaluation of such therapies requires a simple method for the assessment of kidney function in a uremic large animal model. Currently, no simple method is available to measure glomerular filtration rate (GFR) in a uremic large animal with a body weight similar to humans. Glomerular filtration rate (GFR) is considered as the best indicator of (residual) kidney function and it is an important biomarker in clinical and pre-clinical drug development, as kidney function is a determinant of the pharmacokinetics of many drugs. The use of a large animal model for this purpose is important because such animals tolerate frequent blood sampling, which allows repetitive measurements and thus assessment of plasma clearance (CL) as well as adequate monitoring of efficacy and safety. Goats seem particularly suitable for the evaluation of novel hemodialysis therapies since these animals are docile, have easily accessible neck veins and have body weights (70-90 kg) and distribution volumes comparable with humans [3]. In addition, pigs were used for the preclinical evaluation of novel therapies for CKD as these animals have similarities to humans in terms of bodyweight (and distribution volume) and renal anatomy and physiology [4-7].

Regulatory agencies, such as the European Medicines Agency (EMA), recommend that during drug development actual GFR is measured (mGFR) at least once [8,9]. GFR can be measured after infusion of an exogenous substance that is freely filtered by glomeruli, and that is not secreted, reabsorbed, synthesized or metabolized by the kidney. The gold standard for mGFR is inulin clearance [10], which requires a continuous infusion of inulin for several hours, a careful assessment of urinary inulin excretion and repeated blood sampling to monitor inulin steady-state plasma concentrations. This method is cumbersome, time-consuming and expensive. Moreover, the production of inulin has recently been discontinued.

Alternative markers for measurement of GFR are available that can be administered as a single bolus injection, including ${ }^{51} \mathrm{Cr}$-EDTA, ${ }^{99 \mathrm{~m}} \mathrm{Tc}-\mathrm{DTPA}$, iothalamate and iohexol [10]. The mGFR derived from renal excretion of ${ }^{51} \mathrm{Cr}$-EDTA and ${ }^{99 \mathrm{~m}} \mathrm{Tc}$-DTPA are reliable methods to determine mGFR, but the use and storage of radioactive agents are restricted to nuclear facilities, which is relatively costly and impractical. Iohexol, a non-isotopic contrast agent, is currently the most widely used alternative marker for GFR measurement in humans due to its ease of use and favorable safety profile and physicochemical properties (molecular weight: $821 \mathrm{Da}$, protein binding: $1.5 \%$, no renal secretion, metabolism or -reabsorption, negligible extra-renal clearance) [11]. Iohexol plasma clearance is calculated by dividing the administered iohexol dose by the area under the plasma iohexol disappearance curve (AUC) using non-compartmental or compartmental models and therefore accurately predicts GFR [10]. Following intravenous administration of iohexol, the plasma concentrationtime profile of iohexol is characterized by a three-exponential curve; a rapid distribution phase, followed by a second slow distribution phase and a constant elimination phase. To accurately measure the plasma clearance of iohexol, frequent blood sampling is required to fully capture the iohexol plasma disappearance curve. To reduce the need for frequent blood sampling after bolus injection, many mathematical corrections were reported to calculate iohexol clearance based on linear regression using only two or three iohexol plasma concentration measurements in the elimination phase, e.g., from $180 \mathrm{~min}$ after bolus iohexol administration onwards [11]. In humans, the method developed by BröchnerMortensen is most widely applied [11,12], where the clearance is calculated based on the linear regression during the constant elimination phase after which a formula is applied to correct for the lacking distribution phases. By this approach, estimating iohexol plasma clearance after a single bolus injection of iohexol and limited blood sampling is relatively easy to perform, less time consuming, reduces total blood sampling volume and eliminates the need for an indwelling urinary catheter as compared to renal clearance methods that require continuous infusion of an exogenous marker and urine collection. 
However, no simple method, such as the Bröchner-Mortensen formula, is available for the measurement of GFR in goats and pigs. Therefore, the primary aim of this study was to develop a simple method to measure GFR repeatedly by plasma clearance in goats and pigs with normal and impaired kidney function after a single bolus injection of iohexol.

\section{Materials and Methods}

\subsection{Animals}

White adult goats (Capra aegagrus hircus) $(n=3)$ weighing $48-84 \mathrm{~kg}$ were obtained from V.O.F. de Römer (Heythuysen, The Netherlands). Goats were screened for caprine arthritis encephalitis, caseous Lymphadenitis, paratuberculosis, and bovine virus diarrhoea, and vaccinated against Q-fever. Clinically healthy female pigs (Topigs Norsvin; $n=5$ ) weighing $34-80 \mathrm{~kg}$ were obtained from Van Beek SPF varkens B.V. (Lelystad, the Netherlands). Pigs were screened for several common viral diseases (pseudorabies, porcine reproductive and respiratory syndrome, classical swine fever, African swine fever, transmissible gastroenteritis, influenza, porcine epidemic diarrhoea, porcine delta coronovirus, rotavirus, swine vesicular disease), bacterial infections (Mycoplasma hyopneumoniae, Actinobacillus pleuropneumonia, brachyspira hyodysenteriae, leptospira, Pasteurella multocida, brucella, MRSA, salmonella) and ectoparasites. After placement of a jugular line, animals were housed indoors in individual cages to prevent dislodgement of the line by other animals. Otherwise, animals were housed in groups. The temperature of the animal room was maintained between 18 and $21^{\circ} \mathrm{C}$ and artificial lighting was provided. Goats were offered $300 \mathrm{~g}$ of dry feed (Kasper Faunafood, Woerden, The Netherlands) per day and pigs $1500 \mathrm{~g}$ of dry feed (9050 Maintenance diet for minipig, rich in crude fibre, Altromin International, Lage, Germany). Hay for goats, straw as bedding for both pigs and goats and water were provided ad libitum.

\subsection{Experimental Design}

GFR was measured in $n=3$ goats (age, range: $6-24$ months) and $n=5$ pigs (3-8 months) before and after the induction of CKD within the same animal ( 2 measurements in $n=2$ goats and 10 measurements in $n=4$ pigs before induction of CKD, and 11 measurements in $n=3$ goats and 13 measurements in $n=5$ pigs afterwards). Uremia was established by embolization of (branches) of the renal artery using polyvinyl alcohol particles, an accepted method [13] aiming for embolization of $\sim 80 \%$ of one kidney and complete embolization of the contralateral kidney. GFR measurements after embolization were performed once plasma urea and creatinine values had stabilized. Repeated experiments in the same animal before and after the induction of kidney failure were performed within a six-week time period to reduce intra-subject variation in GFR. In one goat, a three-month interval was present between the first and last GFR measurement during which clinical condition and plasma urea and creatinine concentrations were stable.

At least one day prior to GFR measurement, an indwelling venous catheter (silicone catheter $7 \mathrm{Fr}, 60 \mathrm{~cm}, 2$ moveable beads with injection cap, Access technologies, Chicago, IL, USA) was placed in the internal jugular vein under general anesthesia (premedication: midazolam $0.7 \mathrm{mg} / \mathrm{kg}$ i.m., ketamine $13 \mathrm{mg} / \mathrm{kg}$ i.m. and atropine sulphate $0.05 \mathrm{mg} / \mathrm{kg}$ i.m.; induction and maintenance: propofol $3 \mathrm{mg} / \mathrm{kg}$ i.v. and $3.5 \mathrm{mg} / \mathrm{kg} / \mathrm{h}$, respectively, and remifentanil $30 \mu \mathrm{g} / \mathrm{kg} / \mathrm{h}$ i.v.) for iohexol administration and repeated blood withdrawal. Animals were awake during experiments. A single intravenous bolus of $1500 \mathrm{mg}$ iohexol ( $5 \mathrm{~mL}$, Omnipaque $300 \mathrm{mg} / \mathrm{mL}$, GE Healthcare, Machelen, Belgium) was administered followed by flushing with sodium chloride $0.9 \%$. A $3.5 \mathrm{~mL}$ venous blood sample was collected in a K2 EDTA tube with a gel separator (Vacuette ${ }^{\circledR}$, Greiner Bio-One, Alphen aan den Rijn, The Netherlands) before bolus administration and after 5, 10, 20, 30, 60, 120, $180,240,300,360,480$ and $1440 \mathrm{~min}$ for measurement of iohexol plasma concentrations. Blood samples were centrifuged within $2 \mathrm{~h}$ and plasma was aliquoted and stored at $-80^{\circ} \mathrm{C}$ until analysis. 


\subsection{Laboratory Analyses}

Iohexol plasma concentrations were measured at the University Medical Center Groningen (Groningen, The Netherlands) using liquid chromatography-tandem mass spectrometry (Thermo Scientific Vanquish ${ }^{\mathrm{TM}}$ UPLC system, Thermo Fisher Scientific, Waltham, MA, USA; Thermo Scientific ${ }^{\mathrm{TM}}$ Quantiva $^{\mathrm{TM}}$ tandem quadrupole mass spectrometer, Thermo Fisher Scientific, San Jose, CA, USA) validated for goat and pig EDTA plasma as described previously [14]. In short, $50-\mu \mathrm{L}$ of internal standard dissolved in trichloroacetic acid for deproteinization $\left(10 \mathrm{mg}\right.$ of ${ }^{2} \mathrm{H}_{5}$-iohexol in $50 \mathrm{~mL}$ trichloroacetic acid $10 \%$ ) was added to $100-\mu \mathrm{L}$ of plasma, calibration curve samples and quality controls. The samples were vortexed for $1 \mathrm{~min}$ prior to centrifuging at $9500 \times g$ for $5 \mathrm{~min}$. After precipitation, $10 \mu \mathrm{L}$ of supernatant was diluted 1:100 with water in an autosampler vial. After vortexing for $1 \mathrm{~min}, 40 \mu \mathrm{L}$ was injected into the LC-MS/MS.

\subsection{Statistical Analysis}

To develop the simplified method to calculate iohexol plasma clearance, a correction factor was applied to a one-compartmental model, using iohexol plasma concentrations measured at 180, 240 and $300 \mathrm{~min}$ (i.e., the terminal elimination phase). The correction factor was derived by comparison of iohexol plasma clearance calculated by a one-compartment model with the "gold standard", i.e., a nonlinear mixed effects model that was based on all available iohexol plasma concentrations.

\subsubsection{Nonlinear Mixed Effects Model to Determine Iohexol Clearance}

As a reference method, the gold standard in population pharmacokinetic analyses was applied; a nonlinear mixed effects model (NLME), which was based on all available iohexol plasma concentrations (i.e., before bolus administration and 5, 10, 20, 30, 60, 120, 180, 240, 300,480 and $1440 \mathrm{~min}$ afterwards). A stepwise approach was used to develop a pharmacokinetic model that accurately describes the observed iohexol plasma concentration profiles. Different structural models were explored, including one-, two- and three-compartment models with linear elimination processes. Parameter estimates, e.g., absolute iohexol plasma clearance $\left(\mathrm{CL}_{\mathrm{NLME}}\right)$, volume of distribution $(\mathrm{V})$ and intercompartmental clearance (Q) were estimated using first-order conditional estimation with interaction. Impaired kidney function was assumed as a discrete covariate prior to model development (parameterized as multiplication by $\mathrm{CL}_{\mathrm{NLME}}$ ). Variability in the PK parameters within individuals within one study occasion (i.e., interindividual variability, IIV) and variability in the PK parameters within individuals between study occasions (i.e., interoccasion variability, IOV) were incorporated in the model, assuming a log-normal distribution of the random effects on the model parameters [15]. Additive, proportional and combined residual variability models were tested. Bodyweight and species were explored as covariates that may explain IIV using correlation matrices of the empirical Bayes estimates of the parameters versus potential covariates. Significant covariates $(p<0.05)$ were taken forward in the model development. For the continuous covariate bodyweight, allometric scaling with and without fixed power coefficients was explored. For the discrete covariate species, separate population parameters were estimated (parameterized as multiplication by population parameter). Model selection and evaluation was based on the minimum objective function value (MOFV, using $p<0.05$, e.g., $>3.84$ points decrease in MOFV), standard goodnessof-fit plots, residual standard error (RSE) of the population parameter estimates and the coefficient of variation (CV) of the IIV [16]. From this pharmacokinetic model, individual values of iohexol clearance ( $C L_{\mathrm{NLME}}$ ) were used for further analysis.

\subsubsection{Simplified Method to Determine Iohexol Clearance}

Following intravenous administration of iohexol, the plasma concentration-time profile of iohexol is characterized by a three-exponential curve; a rapid distribution phase, followed by a second slow distribution phase and a constant elimination phase (Figure 1). The intercept $\left(C_{1}\right)$ and slope of the constant elimination phase $\left(b_{1}\right)$ were calculated by 
linear regression in $\mathrm{R}(\operatorname{lm}(\log [$ concentration] time) $)$ using iohexol plasma concentrations measured at 180, 240 and $300 \mathrm{~min}$ (terminal elimination phase, Figure 1a).

The amount of iohexol infused $\left(Q_{\text {inf }}\right)$, the intercept and slope and the terminal elimination phase were used to calculate the clearance assuming a one-compartmental pharmacokinetic disposition model ( $\mathrm{CL}_{1 \mathrm{CMT}}$, Equation (1) [12]).

$$
\mathrm{CL}_{1 \mathrm{CMT}}=\frac{Q_{\text {inf }}}{C_{1} / b_{1}}
$$

The relationship between individual values of $\mathrm{CL}_{\mathrm{NLME}}$ and $\mathrm{CL}_{1 \mathrm{CMT}}$ was explored by linear, non-linear or two-segmented linear relationships where the breakpoint was estimated by the software. The most appropriate relationship was chosen based on goodnessof-fit, numerical diagnostics and Blant-Altman plots. Bias was obtained from the BlandAltman analysis by estimating the mean difference and the standard deviation of the differences between $\mathrm{CL}_{\mathrm{NLME}}$ and $\mathrm{CL}_{1 \mathrm{CMT}}$, and precision $(2 \times$ standard deviation/mean $\times 100 \%)$ was calculated for both methods. Finally, we considered the accuracy within $30 \%$ and $10 \%$, i.e., the percentage of $\mathrm{CL}_{\mathrm{SM}}$ estimates falling within $\pm 30 \%$ (P30) or $\pm 10 \%$ (P10) of $\mathrm{CL}_{\mathrm{NLME}}$. The resulting equation provides a simplified method (SM) to calculate the individual CL as a function of $\mathrm{CL}_{1 \mathrm{CMT}}\left(\mathrm{CL}_{\mathrm{SM}}\right)$.

To calculate mGFR, iohexol plasma clearance was normalized to a standardized body surface area (BSA) of an animal of $70 \mathrm{~kg}$ (Equation (3) for goats, Equation (5) for pigs). The BSA of goats was calculated using previously published methods by Saito (Equation (2)) [17]. For pigs, the BSA was calculated, as described by Kelly (Equation (4)) [18].

$$
\begin{gathered}
\text { BSA }_{\text {SAITO }}=\mathrm{WGT}_{\mathrm{i}}^{0.62} \times 1147.7 / 10000 \\
\mathrm{GFR}_{\text {SAITO }}=\mathrm{CL}_{\mathrm{n}} / \mathrm{BSA}_{\text {SAITO }} \times 1.43 \times 1000
\end{gathered}
$$

where 1.43 is the BSA in $\mathrm{m}^{2}$ for a typical $70 \mathrm{~kg}$ goat.

$$
\begin{gathered}
\mathrm{BSA}_{\text {Kelly }}=\mathrm{WGT}_{\mathrm{i}}^{0.656} \times 734 / 10000 \\
\mathrm{GFR}_{\text {Kelly }}=\mathrm{CL}_{\mathrm{n}} / \mathrm{BSA}_{\text {Kelly }} \times 1.19 \times 1000
\end{gathered}
$$

where 1.19 is the BSA in $\mathrm{m}^{2}$ for a typical $70 \mathrm{~kg}$ pig.

For each animal, mGFR was calculated according to the NLME method and the SM, based on their respective clearances. Bias was obtained from a Bland-Altman analysis and precision was calculated for both methods as were the P30 and P10 for mGFR $\mathrm{SM}_{\mathrm{M}}$.

\subsection{Software}

NLME was performed in NONMEM 7.3 (Icon, Dublin, Ireland) [19]. All data processing, statistical analyses and graphical representations were performed in $\mathrm{R}$ version 3.5.3 (The R Foundation for Statistical Computing, Vienna, Austria) [20], using the tidyverse, segmented, blandr and ggplot2 packages. 

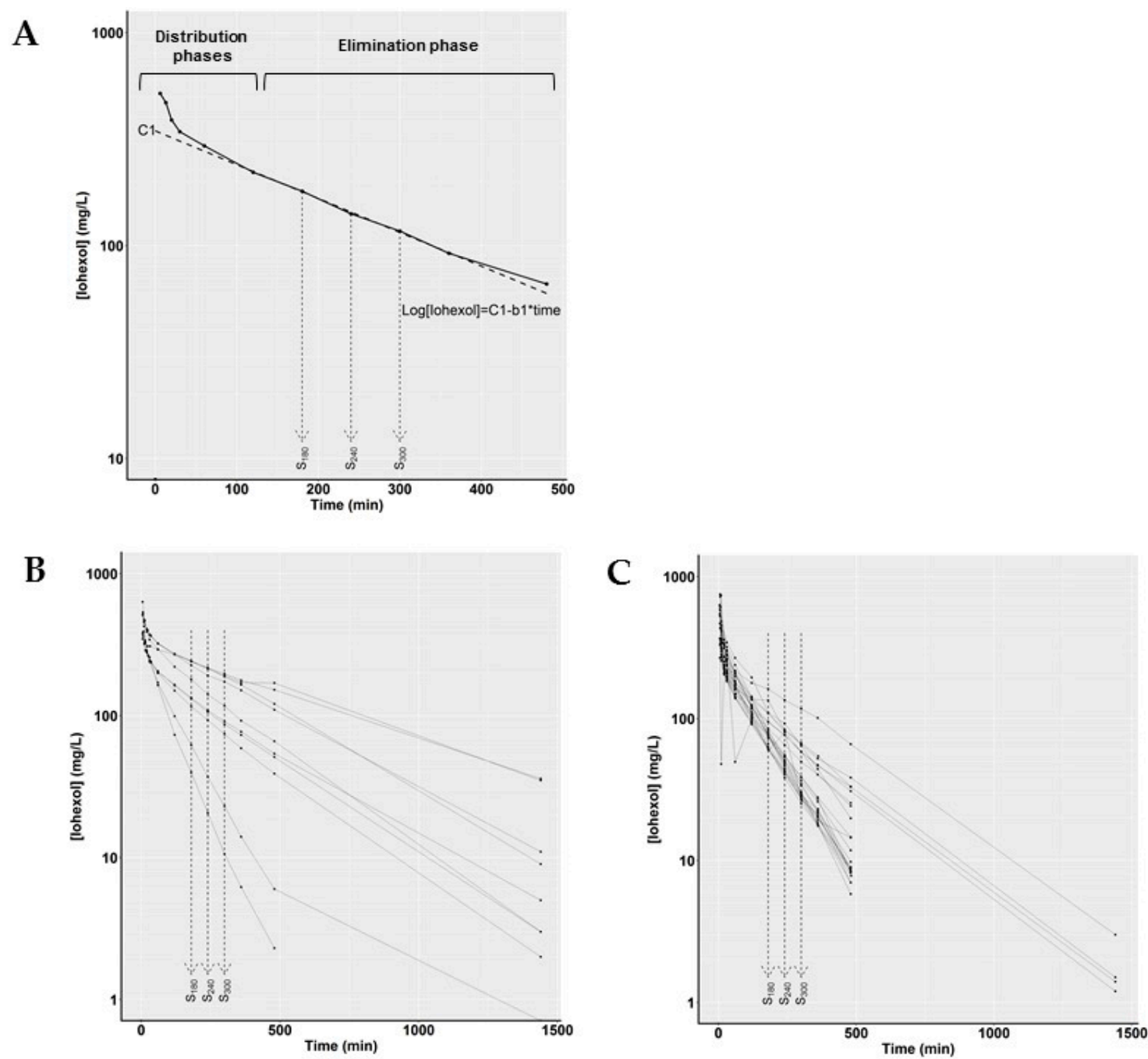

Figure 1. (A) Example of a typical plasma concentration-time profile after bolus administration of iohexol in a uremic goat $(n=1)$. Solid circles represent the iohexol plasma concentrations, the dotted line represents the linear regression (intercept; $C_{1}$, slope; $\left.b_{1}\right)$ over the log-transformed iohexol plasma concentrations taken at sample times 180,240 and 300 min $\left(S_{180}\right.$, $S_{240}$ and $S_{300}$, respectively). (B,C) Plasma concentration-time profiles of all iohexol plasma clearance measurements after bolus administration of iohexol in goats (B) and pigs (C). Circles represent the iohexol plasma concentrations, the dotted lines represent the iohexol plasma concentrations taken at $\mathrm{S}_{180}, \mathrm{~S}_{240}$ and $\mathrm{S}_{300}$, which were used for linear regression analysis according to the simplified method.

\section{Results}

\subsection{Statistical Analysis}

\subsubsection{Data}

In total, 13 iohexol clearance measurements were performed in $n=3$ goats ( 2 measurements in healthy goats, 11 measurements in uremic goats) and 23 measurements were performed in $n=4$ pigs ( 10 measurements in healthy pigs, 13 measurements in uremic pigs). Iohexol plasma concentrations below the lower limit of quantification were excluded for statistical analysis (14 (3\%) of 469 samples in total). One extreme outlier was excluded (iohexol concentration was 11,317 mg/L). During two clearance measurements, maximum iohexol plasma concentration was reached after $20 \mathrm{~min}$ and $180 \mathrm{~min}$, respectively. As this concentration-time profile is illogical after intravenous bolus infusion and suggests extravasation of iohexol, these clearance measurements were excluded from further analysis. Iohexol plasma clearances before and after induction of CKD are presented in Figure 2. 


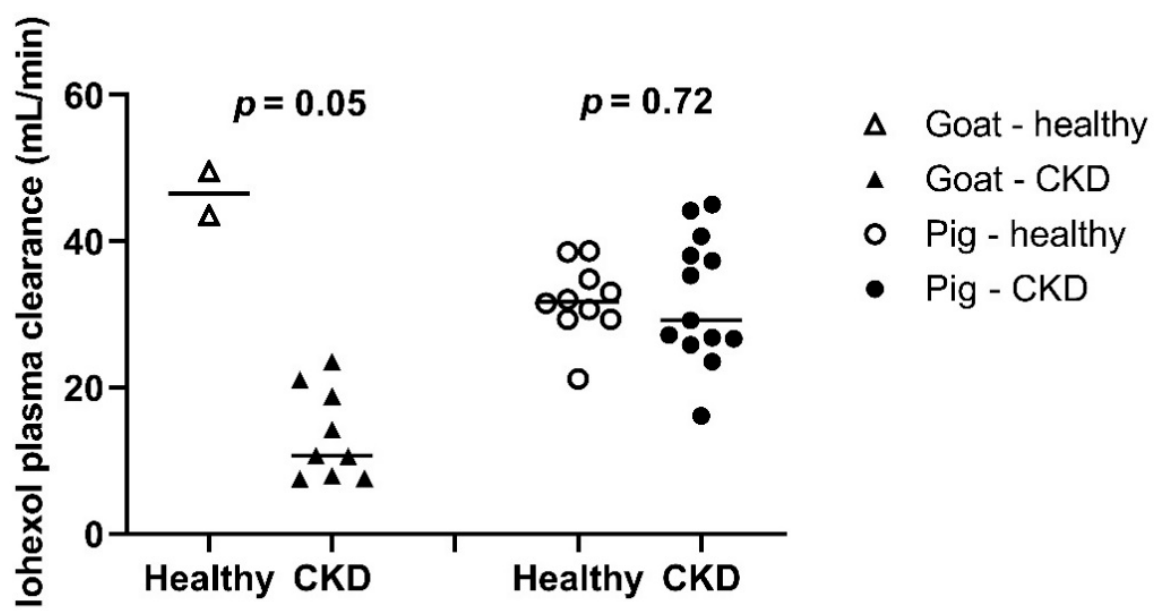

Figure 2. Iohexol plasma clearances after bolus administration of iohexol in goats and pigs (3-8 months of age) before and after induction of CKD within the same animal (2 measurements in $n=2$ goats and 10 measurements in $n=4$ pigs before induction of CKD, and 11 measurements in $n=3$ goats and 13 measurements in $n=5$ pigs afterwards). $p$ values were calculated using a Student's paired t-test.

\subsubsection{Nonlinear Mixed Effects Model to Determine Iohexol Clearance}

In the development of the structural pharmacokinetic model, a three-compartment model $(\mathrm{MVOF}=2811.5)$ proved significantly better at describing the data compared to a one- $(\mathrm{MVOF}=3139.0)$ or two-compartment $(\mathrm{MVOF}=2896.6)$ model. Besides the a priori parameter for impaired kidney function, IOV was identified on CL and IIV on the peripheral volume of distribution. A proportional error model proved best fit for purpose. The incorporation of bodyweight as allometric scaling with fixed power coefficients improved the goodness-of-fit plots. In addition, the scaling of CL between the species improved the model significantly. Bodyweight and species were therefore included in the model as covariates.

In general, the population and individual trend of the data are well captured by the model, both for goats and pigs, as both the population and individual data lie randomly scattered around the line of unity (Figure S1). The goodness-of-fit plots show that the conditional weighted residuals with interaction seem to increase over time, although still within acceptable levels. The population parameter estimates (Table S1) were estimated with high precision as indicated by their low RSE, ranging from 4.6 to $13.5 \%$. Most importantly, the individual iohexol plasma concentration-time profiles were accurately described by the model.

\subsubsection{Simplified Method to Determine Iohexol Clearance}

The relationship between individual values of the $\mathrm{CL}_{\mathrm{NLME}}$ (range; $7.35-46.83 \mathrm{~mL} / \mathrm{min}$ ) and $\mathrm{CL}_{1 \mathrm{CMT}}$ (range; $7.82-57.28 \mathrm{~mL} / \mathrm{min}$ ) was explored by linear, non-linear or segmented linear relationships. The linear regression was statistically significant $(p<0.01)$ with a residual standard error (RSE) of 1.75 on 32 degrees of freedom (DF) and an adjusted $R^{2}$ of 0.976 and Akaike information criterion (AIC) of 138.49. The standardized residuals vs. fitted values ranged from approximately -6 to 3 , although most values lie above 0 . Forcing the linear model through the origin worsened the fit $(p<0.01)$ with a RSE of 2.03 (DF $=33$ ) and AIC of 147.49, despite an improvement of the adjusted $R^{2}$ to 0.995 . The bias in the goodness-of-fit remained. The non-linear model $\left(\mathrm{CL}=\mathrm{ax}-\mathrm{bx}^{2}\right)$ improved the fit $(p<0.01$, Figure 1) to an RSE of $1.67(\mathrm{DF}=32)$ and AIC of 135.23. The standardized residuals vs. fitted values ranged from approximately -2.5 to 2 and most values were now randomly scattered around 0 . The segmented model $(p<0.01)$, with an estimated breakpoint of $23.7 \mathrm{~mL} / \mathrm{min}$, did not outperform the nonlinear model, with an RSE of 1.71 (DF = 30), adjusted $R^{2}$ of 0.98 but a higher AIC of 138.82 . There was no visible improvement in the 
standardized residuals vs. fitted values. The nonlinear model was considered to be most fit for purpose, as there was no apparent improvement using the segmented model, which is also more difficult to interpret and apply. The relationship between $\mathrm{CL}_{1 \mathrm{CMT}}$ and $\mathrm{CL}_{\mathrm{NLME}}$ is depicted in Figure 3.

The formula for the calculation of the plasma clearance according to the simplified method (CL $\left.L_{S M}\right)$ is depicted in Equation (6). The constants 1.006348 and 0.003437 are statistically different from $0(p<0.0001)$ with standard errors of $3.6 \times 10^{-2}$ and $8.4 \times 10^{-4}$. In short, one can now use three blood samples $(t=180, t=240$ and $t=300 \mathrm{~min})$ and equation 1 to estimate $\mathrm{CL} 1_{\mathrm{CMT}}$, and calculate $\mathrm{CL}_{\mathrm{SM}}$ according to Equation (6).

$$
\mathrm{CL}_{\mathrm{SM}}=1.006348 \times \mathrm{CL}_{1 \mathrm{CMT}}-0.003437 \times \mathrm{CL}_{1 \mathrm{CMT}}^{2}
$$

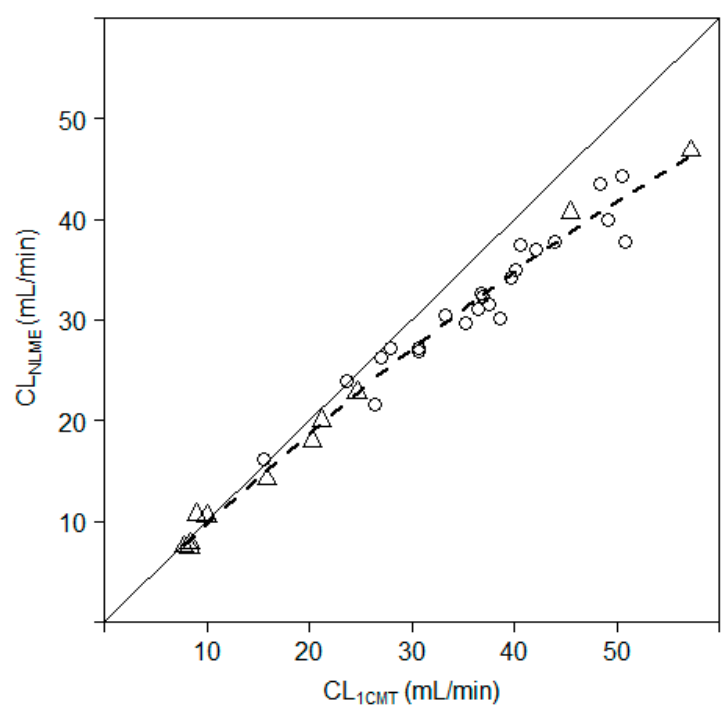

Figure 3. Comparison iohexol plasma clearance based on NLME ( $\mathrm{CL}_{\mathrm{NLME}}$ ) and a one-compartment pool model $\left(\mathrm{CL}_{1 \mathrm{CMT}}\right)$. Dotted line, nonlinear regression line; solid line, line of unity; triangles, goat; circles, pig.

\subsubsection{Evaluation of the Simplified Method versus Reference Method}

Figure 4a shows the values of the measured iohexol plasma clearance based on a nonlinear mixed effects model ( $\mathrm{CL}_{\mathrm{NLME}}$ ) versus the calculated iohexol plasma clearance based on the simplified method $\left(\mathrm{CL}_{\mathrm{SM}}\right.$, range; $\left.7.67-46.37 \mathrm{~mL} / \mathrm{min}\right)$. All values follow the line of unity with a mean $( \pm \mathrm{SD}) \mathrm{CL}_{\mathrm{NLME}} / \mathrm{CL}_{\mathrm{SM}}$ ratio of $1.00 \pm 0.07$. Similarly, BlandAltman analysis (Figure $4 \mathrm{~b}$ ) showed that the mean bias $( \pm \mathrm{SD})$ is $0.03 \pm 1.64$ with upper and lower limits of agreement of \pm 3.2 , resulting in a precision of agreement between the two methods of $11.6 \%$. The precision of the $C_{\text {NLME }}$ and $C_{S M}$ were $80.9 \%$ and $80.7 \%$, respectively (Table 1 ). The $\mathrm{P} 30$ and $\mathrm{P} 10$ of $\mathrm{CL}_{\mathrm{SM}}$ were $53 \%$ and $12 \%$, respectively. The data are randomly scattered around 0 , indicating no consistent bias of one method versus the other. There is no evidence of a species-dependent bias. 

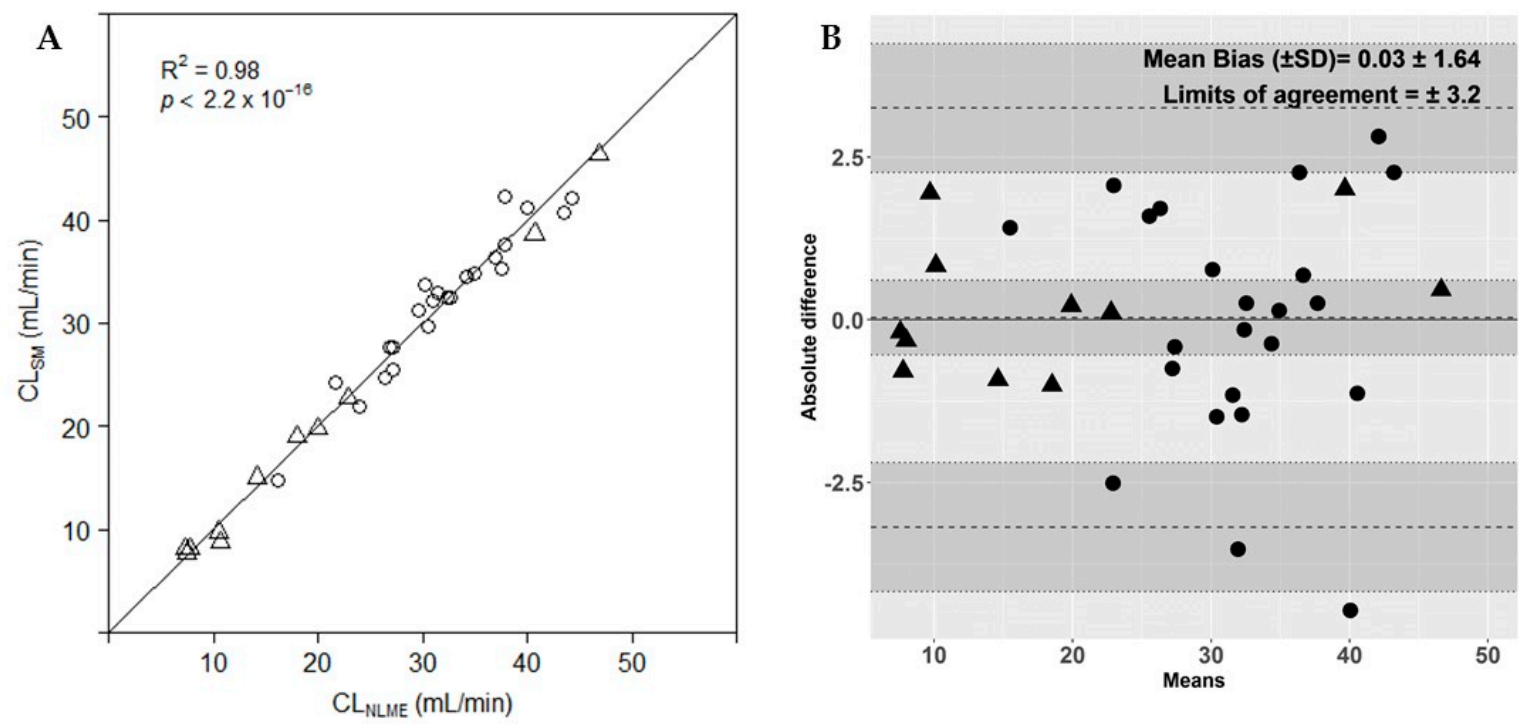

Figure 4. (A) Comparison of the measured iohexol plasma clearance based on a nonlinear mixed effects model (CLNLME) and calculated iohexol plasma clearance based on the simplified method (CLSM) in goats (triangles) and pigs (circles). Solid line, line of unity. (B) Bland-Altman plot of agreement between iohexol plasma clearance determined by a nonlinear mixed effects model and by the simplified method for goats (triangles) and pigs (circles). Dotted lines represent bias, upper and lower limits of agreement. Grey shaded areas represent the $95 \%$ confidence intervals of the bias, upper and lower limits of agreement.

Table 1. Precision and accuracy of iohexol plasma clearance and mGFR calculated by a nonlinear mixed effects model and the simplified method.

\begin{tabular}{ccccc}
\hline \multirow{2}{*}{ Parameter } & Method & Precision (\%) & \multicolumn{2}{c}{ Accuracy (\%) } \\
\cline { 4 - 5 } & & & P30 * & P10 * \\
\hline \multirow{2}{*}{ CL } & NLME & 80.9 & 53 & 12 \\
& SM & 80.7 & & \multirow{2}{*}{24} \\
\hline \multirow{2}{*}{ mGFR } & NLME & 49.2 & 71 & 24 \\
& SM & 53.6 &
\end{tabular}

CL; iohexol plasma clearance, mGFR, measured glomerular filtration rate, NLME; nonlinear mixed effects model SM; simplified method. Precision was calculated as: $2 \times$ standard deviation/mean $\times 100 \%$. ${ }^{*}$ The percentage of SM estimates falling within $\pm 30 \%$ (P30) or $\pm 10 \%$ (P10) of CL calculated by NLME.

\subsection{5. mGFR Results}

Figure 5 a shows the values of the $\mathrm{mGFR}$ based on $\mathrm{CL}_{\mathrm{NLME}}$ (range; $\left.16.6-46.3 \mathrm{~mL} / \mathrm{min} / \mathrm{BSA}\right)$ versus the $\mathrm{mGFR}$ based on $\mathrm{CL}_{\mathrm{SM}}$ (range; $15.2-50.7 \mathrm{~mL} / \mathrm{min} / \mathrm{BSA}$ ), assuming a BSA of $1.43 \mathrm{~m}^{2}$ in goats and $1.19 \mathrm{~m}^{2}$ in pigs. All values follow the line of unity with a ratio of $1.00 \pm 0.06$. The Bland-Altman analysis (Figure $5 \mathrm{~b}$ ) showed that there is a bias of $-0.25 \pm 2.24$ with upper and lower limits of agreement of \pm 4.4 , resulting in a percentage of error between the two methods of $12.5 \%$. The precisions of mGFR based on $\mathrm{CL}_{\mathrm{NLME}}$ and mGFR based on $\mathrm{CL}_{\mathrm{SM}}$ separately were $49.2 \%$ and $53.6 \%$. The P30 and P10 of $\mathrm{CL}_{\mathrm{SM}}$ were $71 \%$ and $24 \%$. Again, there is no specific bias towards species.

There are no data in the 95 confidence interval of the upper limit of agreement, indicating a slight bias of one approach versus the other, where the simplified method seems to slightly overestimate the mGFR in 2 goats and 3 pigs. 

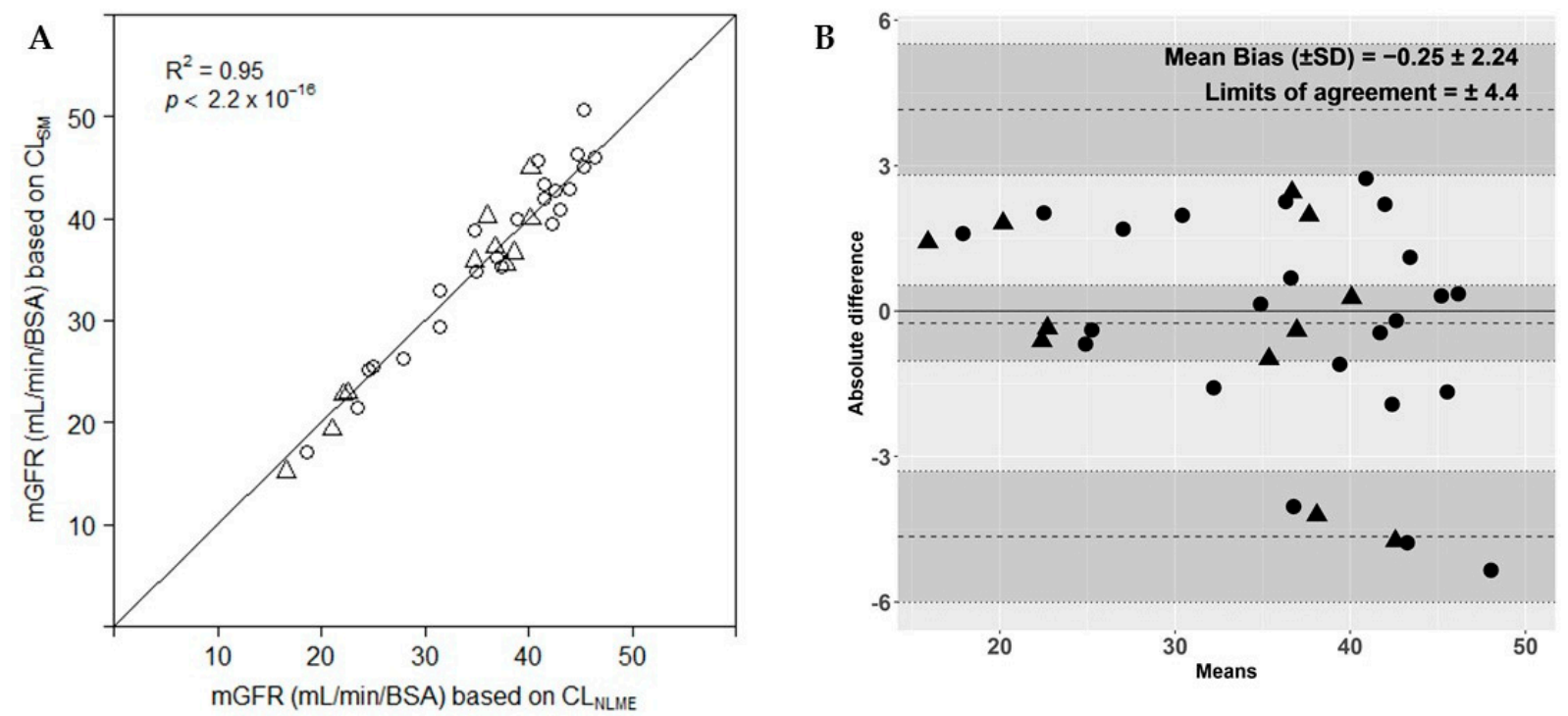

Figure 5. (A): Comparison of measured glomerular filtration rate (mGFR) based on clearance determined by a nonlinear mixed effects model ( $\mathrm{CL}_{\mathrm{NLME}}$ ) vs. the mGFR based on clearance determined by the simplified method (CLSM). All GFR values were adjusted for body surface area (BSA), assuming a BSA of $1.43 \mathrm{~m}^{2}$ in goats and $1.19 \mathrm{~m}^{2}$ in pigs. Solid line, line of unity; triangles, goat; circles, pig. (B): Bland-Altman plot of agreement between measured glomerular filtration rate (mGFR) based on plasma clearance determined by a nonlinear mixed effects model ( $\mathrm{CL}_{\mathrm{NLME}}$ ) and mGFR based on clearance determined by the simplified method ( $\mathrm{CL}_{\mathrm{SM}}$ ) for goats (triangles) and pigs (circles). Dotted lines represent bias, upper and lower limits of agreement. Grey shaded areas represent the $95 \%$ confidence intervals of the bias, upper and lower limits of agreement.

\section{Discussion}

A simple method for measurement of glomerular filtration rate (GFR) in healthy and uremic goats and pigs was developed that is based on iohexol plasma clearance after a single bolus administration. This simplified method was developed by application of a correction factor to a one-compartmental model, based on the terminal elimination phase, to calculate iohexol plasma clearance. The correction factor was derived by comparison of the one-compartment model with the "true clearance" based on a nonlinear mixed effects model.

Our method has several advantages as compared to plasma clearance determined by continuous inulin infusion and measurement of urinary inulin excretion, which currently is the gold standard for GFR measurement [10]. After bolus administration, only three venous blood samples are required at 180,240 and $300 \mathrm{~min}$ without the need for continuous infusion of an exogenous marker, frequent blood sampling during steady-state plasma concentrations or timed urine collections. Moreover, the simplified method, based on sparse sampling, uses standard linear regression, which can be performed in any statistical package, whereas NLME, which is based on more frequent sampling, requires specialized software and personnel. Therefore, this method is easy to perform, less time-consuming and reduces animal discomfort.

To the best of our knowledge, we are the first to develop a method for GFR measurement based on iohexol plasma clearance in goats and pigs. Few studies have measured GFR in pigs using exogenous markers other than inulin [7,21-25] and to our knowledge, there are no such reports in goats. Similar to our study, Luis-Lima has developed a method for calculation of GFR based on blood sampling after bolus injection of iohexol in healthy swine weighing $\sim 150 \mathrm{~kg}$ [21]. However, their model has not been validated in animals with impaired kidney function or a body weight similar to humans and requires six blood samples up to $420 \mathrm{~min}$, whereas our method relies on only three blood measurements between 180 and $300 \mathrm{~min}$. One study measured GFR in pigs with impaired kidney function by quantification of urinary iohexol excretion [25]. However, urinary excretion methods 
are not ideal as urine collection in animals is cumbersome, prone to errors and may require temporary sedation of animals for insertion of an indwelling urinary catheter. In goats, only inulin excretion methods were used [26-32]. In sheep, which resemble goats, a method for measurement of iohexol plasma clearance was developed, which is based on seven blood samples, whereas our method requires only three blood samples [31].

The Bland-Altman plot showed good agreement between the observed CL (CLNLME) and the CL calculated by the simplified method (CLSM). The mean ratio was nearly 1 , as expected since the simplified method is derived from NLME modeling. However, the simplified method does not take into account interindividual or intra-occasion variability. As such, the standard deviation of the ratio is more important, which was low. This indicates that the simplified method can be used to estimate the true individual clearance in both goats and pigs. Interestingly, when adjusting CL for BSA, a slight bias between the methods occurred, particularly in the higher mGFR range. This may be caused by proportional inaccuracies in the measurement of body weight or the methods for the calculation of BSA.

This study has limitations. First, the relatively limited number of animals may have caused bias by the inclusion of species as a covariate in the model. Second, extrarenal clearance of iohexol was not measured and could theoretically have resulted in an overestimation of GFR. However, Frennby et al. found that extrarenal excretion of iohexol is very limited in anephric pigs $(0.087 \mathrm{~mL} / \mathrm{min} / \mathrm{kg})$ [33]. Therefore, we do not expect that extrarenal clearance has influenced the conclusions of the present study.

\section{Conclusion}

In conclusion, a simple, accurate and minimally invasive method was developed to measure GFR based on plasma clearance of iohexol in healthy and uremic goats and pigs. This method could be used for monitoring GFR in animals during a preclinical drug or medical device development for the treatment of CKD.

Supplementary Materials: The following are available online at https://www.mdpi.com/article/10 .3390/biology10060461/s1, Figure S1: Goodness-of-fit plots population PK model, Table S1: Population parameter estimates of the pharmacokinetic iohexol model.

Author Contributions: Conceptualization, M.K.v.G., J.S. and K.G.F.G.; Data curation, M.K.v.G. and J.S.; Formal analysis, M.K.v.G. and J.S.; Funding acquisition, K.G.F.G.; Investigation, M.K.v.G., J.S. and K.R.D.V.; Methodology, M.K.v.G., J.S., T.T.P. and K.G.F.G.; Project administration, K.G.F.G.; Resources, K.G.F.G.; Supervision, K.G.F.G.; Validation, M.K.v.G. and J.S.; Visualization, M.K.v.G. and J.S.; Writing—original draft, M.K.v.G. and J.S.; Writing—review and editing, T.T.P., K.R.D.V., J.A.J., M.C.V. and K.G.F.G. All authors have read and agreed to the published version of the manuscript.

Funding: This study was funded by the European Union (WEAKID, Horizon 2020 research and innovation program, grant agreement no. 733169) and by the Dutch Kidney Foundation.

Institutional Review Board Statement: The study was approved by the Animal Experiments Committee (Utrecht, The Netherlands) and performed in accordance with national guidelines for the care and handling of animals (AVD115002015226, 1-10-2015).

Informed Consent Statement: Not applicable.

Data Availability Statement: Data is contained within the article or Supplementary Material.

Conflicts of Interest: The authors declare no conflict of interest.

\section{References}

1. Stevens, P.E.; Levin, A. Evaluation and management of chronic kidney disease: Synopsis of the kidney disease: Improving global outcomes 2012 clinical practice guideline. Ann. Intern. Med. 2013, 158, 825-830. [CrossRef] [PubMed]

2. Saran, R.; Robinson, B.; Abbott, K.C.; Bragg-Gresham, J.; Chen, X.; Gipson, D.; Gu, H.; Hirth, R.A.; Hutton, D.; Jin, Y.; et al. US Renal Data System 2019 Annual Data Report: Epidemiology of Kidney Disease in the United States. Am. J. Kidney Dis. 2020, 75, A6-A7. [CrossRef] 
3. Wester, M.; Gerritsen, K.G.; Simonis, F.; Boer, W.H.; Hazenbrink, D.H.; Vaessen, K.R.; Verhaar, M.C.; Joles, J.A. A regenerable potassium and phosphate sorbent system to enhance dialysis efficacy and device portability: A study in awake goats. Nephrol. Dial. Transplant. 2017, 32, 951-959. [CrossRef]

4. Giraud, S.; Favreau, F.; Chatauret, N.; Thuillier, R.; Maiga, S.; Hauet, T. Contribution of large pig for renal ischemia-reperfusion and transplantation studies: The preclinical model. J. Biomed. Biotechnol. 2011, 2011, 532127. [CrossRef] [PubMed]

5. Chade, A.R.; Williams, M.L.; Engel, J.; Guise, E.; Harvey, T.W. A translational model of chronic kidney disease in swine. Am. J. Physiol. Renal Physiol. 2018, 315, F364-F373. [CrossRef]

6. Misra, S. The Porcine Remnant Kidney Model of Chronic Renal Insufficiency. J. Surg. Res. 2006, 135, 370-379. [CrossRef]

7. Robbins, M.E.C.; Robinson, M.; Rezvani, M.; Golding, S.J.; Hopewell, J.W. The response of the pig kidney to the combined effects of cisplatin and unilateral renal irradiation. Radiother. Oncol. 1988, 11, 271-278. [CrossRef]

8. Boele-Schutte, E.; Gansevoort, R.T. Measured GFR: Not a gold, but a gold-plated standard. Nephrol. Dial. Transplant. 2017, 32, ii180-ii184. [CrossRef] [PubMed]

9. Committee for Medicinal Products for Human Use (CHMP). Guideline on the Evaluation of the Pharmacokinetics of Medicinal Products in Patients with Decreased Renal Function; European Medicines Agency: London, UK, 2014.

10. Soveri, I.; Berg, U.B.; Bjork, J.; Elinder, C.G.; Grubb, A.; Mejare, I.; Sterner, G.; Back, S.E.; Group, S.G.R. Measuring GFR: A systematic review. Am. J. Kidney Dis. 2014, 64, 411-424. [CrossRef] [PubMed]

11. Delanaye, P.; Ebert, N.; Melsom, T.; Gaspari, F.; Mariat, C.; Cavalier, E.; Bjork, J.; Christensson, A.; Nyman, U.; Porrini, E.; et al. Iohexol plasma clearance for measuring glomerular filtration rate in clinical practice and research: A review. Part 1: How to measure glomerular filtration rate with iohexol? Clin. Kidney J. 2016, 9, 682-699. [CrossRef]

12. Brochner-Mortensen, J. A simple method for the determination of glomerular filtration rate. Scand. J. Clin. Lab. Investig. 1972, 30, 271-274. [CrossRef] [PubMed]

13. Belis, J.A.; Horton, J.A. Renal artery embolization with polyvinyl alcohol foam particles. Urology 1982, 19, 224-227. [CrossRef]

14. Stevens, J.; Wessels, M.A.; Roggeveld, J.; Koster, R.A.; Dekkers, C.C.; van Gelder, M.K.; Gansevoort, R.T.; Heerspink, H.J.; Touw, D.J. UHPLC-MS/MS method for iohexol determination in human EDTA and lithium-heparin plasma, human urine and in goatand pig EDTA plasma. Bioanalysis 2020, 12, 981-990. [CrossRef]

15. Karlsson, M.O.; Sheiner, L.B. The importance of modeling interoccasion variability in population pharmacokinetic analyses. J. Pharm. Biopharm. 1993, 21, 735-750. [CrossRef] [PubMed]

16. Mould, D.R.; Upton, R.N. Basic concepts in population modeling, simulation, and model-based drug development-part 2: Introduction to pharmacokinetic modeling methods. CPT Pharmacomet. Syst. Pharmacol. 2013, 2, e38. [CrossRef]

17. Saito, M.; Tasaki, I.; Annaka, A. Studies on the metabolism of dairy goat. J.-Stage 1954, 25, 25-29.

18. Swindle, M.M. Swine in the Laboratory: Surgery, Anesthesia, Imaging and Experimental Techniques, 2nd ed.; CRC Press: Boca Raton, FL, USA, 2007.

19. Beal, S.L.; Sheiner, L.B.; Boeckmann, A.J.; Bauer, R.J. (Eds.) NONMEM 7.3.0 Users Guides (1989-2013); ICON Development Solutions: Hanover, MD, USA, 2013.

20. R Core Team. R: A Language and Environment for Statistical Computing; R Foundation for Statistical Computing: Vienna, Austria, 2010.

21. Luis-Lima, S.; Garcia-Contreras, C.; Vazquez-Gomez, M.; Astiz, S.; Carrara, F.; Gaspari, F.; Negrin-Mena, N.; Jimenez-Sosa, A.; Jimenez-Hernandez, H.; Gonzalez-Bulnes, A.; et al. A Simple Method to Measure Renal Function in Swine by the Plasma Clearance of Iohexol. Int. J. Mol. Sci. 2018, 19, 232. [CrossRef]

22. Lodrup, A.B.; Karstoft, K.; Dissing, T.H.; Nyengaard, J.R.; Pedersen, M. The association between renal function and structural parameters: A pig study. BMC Nephrol. 2008, 9, 18. [CrossRef]

23. Gasthuys, E.; Devreese, M.; Millecam, J.; Sys, S.; Vanderperren, K.; Delanghe, J.; Vande Walle, J.; Heyndrickx, M.; Croubels, S. Postnatal Maturation of the Glomerular Filtration Rate in Conventional Growing Piglets As Potential Juvenile Animal Model for Preclinical Pharmaceutical Research. Front. Pharmacol. 2017, 8, 431. [CrossRef]

24. Palnaes Hansen, C.; Bie, P.; Stadil, F. Assessment of renal function by 51Cr-EDTA and endogenous creatinine clearances in the pig. Acta Physiol. Scand. 1997, 161, 253-260. [CrossRef] [PubMed]

25. Frennby, B.; Sterner, G.; Almen, T.; Chai, C.M.; Jonsson, B.A.; Mansson, S. Clearance of iohexol, 51Cr-EDTA and endogenous creatinine for determination of glomerular filtration rate in pigs with reduced renal function: A comparison between different clearance techniques. Scand. J. Clin. Lab. Investig. 1997, 57, 241-252. [CrossRef] [PubMed]

26. Skotnicka, E.; Muszczynski, Z.; Dudzinska, W.; Suska, M. A review of the renal system and diurnal variations of renal activity in livestock. Ir. Vet. J. 2007, 60, 161-168. [CrossRef] [PubMed]

27. Vogel, G. Beitrage zur Kenntius der Nierenphysiologic einiger Haussaugetiere [Contribution to the knowledge on kidney physiology in some domestic mammals]. Zentl Vet. Med. Beiheft 1962, 3. (In German) [CrossRef]

28. Ketz, H.A. Vergleichende Betrachtungen zur Nierenfunktion bei den Haustieren [Comparative studies on kidney function in domestic animals]. Arch. Exp. Vet. Med. 1960, 14, 411-419. (In German)

29. Muszczynski, Z.; Skotnicka, E.; Jankowiak, D. Diurnal variations of renal activity in goats. Turk. J. Vet. Anim. Sci. 2015, 39, 455-464. [CrossRef]

30. Ladd, M.; Liddle, L.; Gagnon, J.A.; Clarke, R.W. Glomerular and tubular functions in sheep and goats. J. Appl. Physiol. 1957, 10, 249-255. [CrossRef] 
31. Luis-Lima, S.; Mas-Sanmartin, C.; Rodriguez-Rodriguez, A.E.; Porrini, E.; Ortiz, A.; Gaspari, F.; Diaz-Martin, L.; Asberg, A.; Jenssen, T.; Jimenez-Sosa, A.; et al. A Simplified Iohexol-Based Method to Measure Renal Function in Sheep Models of Renal Disease. Biology 2020, 9, 259. [CrossRef]

32. Nesje, M.; Flaoyen, A.; Moe, L. Estimation of glomerular filtration rate in normal sheep by the disappearance of iohexol from serum. Vet. Res. Commun. 1997, 21, 29-35. [CrossRef]

33. Frennby, B.; Sterner, G.; Almen, T.; Chai, C.M.; Jonsson, B.A.; Mansson, S. Extrarenal plasma clearance of iohexol, chromium-51ethylenediaminetetraacetic acid, and inulin in anephric pigs. Acad. Radiol. 1996, 3, 145-153. [CrossRef] 